\title{
Melittopalynological studies of Apis dorsata honey samples from Kolar District, Karnataka, India
}

\author{
Gopal, T.C. \\ Department of Botany, J.S.S Arts, Science and Commerce College, Gokak (Karnataka), \\ India \\ Email: gopaltcgokak@gmail.com
}

\section{Article Info}

https://doi.org/10.31018/

jans.v13i2.2189

Received: April 3, 2021

Revised: May 26, 2021

Accepted: May 29, 2021

\section{How to Cite}

Gopal, T. C. (2021). Melittopalynological studies of Apis dorsata honey samples from Kolar District, Karnataka, India. Journal of Applied and Natural Science, 13(2), 654 - 667. https://doi.org/10.31018/jans.v13i2.2189

\begin{abstract}
Honeybees, while foraging for nectar on flowers, also gather some pollen which retains in the honey even after extraction. Pollen grains are the essential tools in the analysis of honey. The aim of the present report was to find the Apis dorsata honey floral resources in Kolar district state Karnataka. In the present study, the pollen content of $28 \mathrm{~A}$. dorsata honey samples were collected from 5 different locations of Kolar district, Karnataka, India. Samples were subjected to Melittopalynological studies to identify their honey plant resources and colour, optic density and collection places were documented. A wide variety of pollen types represent their plant sources and their frequency classes were recognized in each honey sample. Among 28 honey samples analysed, 10 samples were identified as multifloral, 18 unifloral with predominant pollen types such as Syzygium cumini, Pongamia pinnata, Eucalyptus sp, Guizotia abyssinica, Psidium guajava and Coriandrum sativum, each count was found above $45 \%$. Pollen spectra indicated a total of 56 pollen types belonging to 27 plant families. Fabaceae was represented as the largest family with 14 species contributing honey production. Among the habit, tree was dominant with $51.78 \%$, followed by herbs $(32.14 \%)$ and shrubs (16.07\%). The economic importance of identified plants with apiculture importance was categorized as medicinal, ornamental, vegetable, timber and oil yielding, weeds, fruits and nuts. $A$. dorsata depends on wild trees and cultivated plants bloom throughout the year as pollen and nectar source. From the results, it is evident that there is a lot of potential in establishing beekeeping industries in the study area.
\end{abstract}

Keywords: Apis dorsata, Kolar district, Melittopalynology, Multifloral, Unifloral

\section{INTRODUCTION}

Melittopalynology is an applied branch of Palynology deals with the microscopic analysis of pollen grains in honey. Honeybees depend on plants for pollen and nectar. In turn bees provide pollination services to a wide variety of flowering plants. Honey contains pollen grains, which are collected by honeybees while foraging the flowers for nectar. The microscopic analysis of pollen is a standard method to identify the abundance of nectar sources, distribution, geographical and botanical origin, and honey adulteration in a given area (Silva and Santos, 2014). Knowledge of floral diversity of apicultural importance is a prerequisite for beekeepers to undertake migratory beekeeping to increasing honey production and pollination (Singh et al., 2016). Beekeeping provides self-employment to the farmers and tribes to generate income. Honeybees naturally produce honey from the nectar of plants. It is widely consumed as a health food product worldwide, but adulter- ation and the false labelling of honey are common problems in many countries (Sajwani et al., 2007; Louveaux et al., 1978). In this context, Melittopalynology plays an important role in ascertaining honey's botanical and geographical origins by studying the pollen contained in the honey (Anklam, 1998; Oliveira et al., 2010; Ramirez-Arriaga et al., 2011; Upadhyay and Bera, 2012).

In the growth and development of honey bees, nectar is the source of carbohydrates and proteins are provided by pollen (Turner, 1984; Lin et al., 1993). Pollen analyses of honey and bee loads are used to learn honey bee foraging ecology, the habitat and vegetation, habitat composition, changes in honey bee food sources and the geographical region of the hive location (Ramalho and Kleinert-Giovannini, 1986; FellerDemalsy et al., 1989; Barth, 1990; Diaz-Losada et al., 1998; Terrab et al., 2004). Pollen contents of honey samples offer dependable evidence on floral resources of honey along with the relative predilections of bees 
amongst the varied assemblies of plant species flowering synchronously (Deodikar and Thakar, 1953; Deodikar, 1961; Garg, 2006).

Melittopalynological study was introduced over a hundred years ago by several scientists from different part of the world (Maurizio, 1975; Lieux, 1980; Agwu and Akanbi, 1985; Moar, 1985; Deodikar, 1961; Alves and Santos, 2014; Jesus et al., 2015; Majid et. al., 2020). In India, the earliest contribution in this field was by Deodikar and Thakar (1953), Sen and Banerjee (1956) and Novais et al. (2009) characterized pollen in honey samples of Mahabaleshwar hills of Maharastra State and from West Bengal, respectively. Later, VishnuMittre (1958) analyzed the pollen content of honey from Nepal, Kashmir and Lucknow. Recently, several scientists reported the botanical origin of honey in many places of India (Shubharani et al., 2012; Raghunandan and Basavarajappa, 2014; Neha Singh and Chaturvedi, 2016; Manju Sahney et al., 2018). However, no study has been reported from Kolar district, Karnataka. The present study aimed to identify the floral diversity of apicultural importance by melittopalynogical studies of $A$. dorsata honey of Kolar district, Karnataka state.

\section{MATERIALS AND METHODS}

\section{Study area}

The Kolar district is situated in the southeastern part of Karnataka state and called the land of gold, silk and milk. The district lies almost in the central part of peninsular India, which has an immense bearing on its geoclimatic conditions and experiences tropical climatic condition throughout the year. The district is situated between $12^{\prime} 46^{\prime}$ and $13^{\prime}$ '58' north latitude and 77' 21' and 78' $35^{\prime}$ east longitude between Eastern and Western coast and is bound on the West by Bangalore and Tumkur districts, South by North Arcot and Dharmapuri districts of Tamilnadu, East and North by Chittor district of Andhra Pradesh. The district has an area of 8,233 sq $\mathrm{km}$ and occupies $12^{\text {th }}$ place in the state, having $11 \mathrm{Ta}$ luk viz., Bagepalli, Bangarpet, Chikaballapur, Chintamani, Gudibande, Gauribidanur, Kolar, Malur, Mulbagal, Siddlagatta and Srinivasapura (Fig. 1). The district is endowed with a number of hills with peaks of varying heights, particularly in the northern part.

Kolar district falls in the eastern dry agro climatic zone. It experiences a semi-arid climate, characterized by typical monsoon tropical weather with hot summer and mild winter. The average rainfall is around $850 \mathrm{~mm}$ with 55-65 rainy days in Kolar district and the greater portion of the rainfall is from September to November. District experience scanty and erratic rainfall with uneven distribution during monsoon. The large variation in rainfall was noticed from year to year and amongst talk to a larger extent. The main occupation of the people of this district is agriculture; $5 \%$ of the total area in the district is covered by forest, $46 \%$ under cultivation and $28 \%$ is uncultivated area.

\section{Preparation of honey samples}

In the present study, 28 honey samples were collected from the hives of $A$. dorsata from 5 different locations of Kolar district such as Kolar, Srinivasapura, Mulbagal, Malur and Bangarpet during 2017-2019. The Honey samples collected details are documented in Table 1.

\section{Melittopalynological analysis}

The collected honey samples were subjected to pollen analysis to characterize and identify the floral origin, according to the guidelines of Erdtman (1960), Louveaux et al. (1978) and Moore et al. (1991). Ten grams of crude honey sample was dissolved in 20 $\mathrm{ml}$ of warm $\left(40{ }^{\circ} \mathrm{C}\right)$ distilled water and then centrifuged for $10 \mathrm{~min}$ at $2500 \mathrm{rpm}$. The sediment was treated with acetolysis mixture, centrifuged and the supernatant was decanted. The sediment was washed twice with distilled water to remove the debris. A drop of glycerine was added to prevent the sample from drying and gently heated. Five different slides were prepared from each honey sample by adding $50 \mu \mathrm{l}$ of treated sample and cover with a coverslip. The slides were subjected to microscopic study to identify the pollen type by using a Leica DM2500 light microscope. The pollen types present in honey samples were identified by comparison with reference slides and pollen photomicrographs. The pollen types and their number were counted by using Haemocytometer. Based on the percentage of pollen type and its distribution frequency, the honey was categorised into unifloral (one pollen type represented $>45 \%$ ) or multifloral (no pollen type $>45 \%$ ) and also classified to different groups like pre-

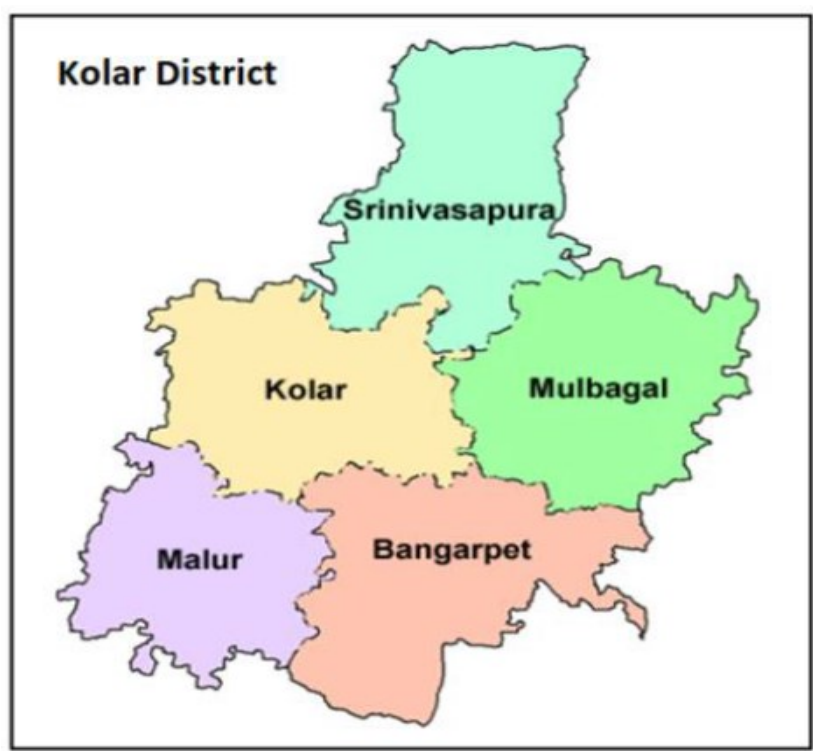

Fig. 1. Map of Kolar district of Karnataka showing honey samples collected areas. 
Gopal, T.C. / J. Appl. \& Nat. Sci. 13(2), 654 - 667 (2021)

Table 1. Details of honey samples collected from Kolar district.

\begin{tabular}{|c|c|c|c|c|}
\hline SI. No. & Code No. & $\begin{array}{l}\text { Place of } \\
\text { Collection }\end{array}$ & $\begin{array}{l}\text { Colour of the } \\
\text { Honey sample }\end{array}$ & $\begin{array}{l}\text { Optical } \\
\text { density }\end{array}$ \\
\hline & $\mathrm{ADH}-1$ & Kolar & Light yellow & 0.383 \\
\hline & $\mathrm{ADH}-2$ & Malur & Yellow & 0.591 \\
\hline & $\mathrm{ADH}-3$ & Srinivasapura & Reddish yellow & 0.209 \\
\hline & $\mathrm{ADH}-4$ & Mulbagal & Reddish yellow & 0.448 \\
\hline & $\mathrm{ADH}-5$ & Bangarpet & Light yellow & 0.490 \\
\hline & $\mathrm{ADH}-6$ & Mulbagal & Dark red & 0.215 \\
\hline & $\mathrm{ADH}-7$ & Malur & Orange & 0.616 \\
\hline & ADH -8 & Kolar & Light yellow & 0.873 \\
\hline & $\mathrm{ADH}-9$ & Srinivasapura & Light yellow & 0.510 \\
\hline & $\mathrm{ADH}-10$ & Mulbagal & Orange & 0.751 \\
\hline & ADH -11 & Kolar & Reddish yellow & 0.473 \\
\hline & ADH -12 & Srinivasapura & Light yellow & 0.807 \\
\hline & ADH -13 & Malur & Reddish yellow & 0.540 \\
\hline & ADH -14 & Malur & Light yellow & 0.359 \\
\hline & ADH -15 & Mulbagal & Light red & 0.803 \\
\hline & ADH -16 & Kolar & Orange & 0.255 \\
\hline & ADH -17 & Malur & Light yellow & 0.996 \\
\hline & ADH -18 & Mulbagal & Reddish yellow & 0.481 \\
\hline & ADH -19 & Kolar & Yellow & 0.712 \\
\hline & ADH -20 & Mulbagal & Dark red & 0.857 \\
\hline & ADH -21 & Bangarpet & Light yellow & 0.988 \\
\hline & ADH -22 & Kolar & Dark yellow & 0.954 \\
\hline & ADH -23 & Srinivasapura & Light yellow & 0.470 \\
\hline & ADH -24 & Kolar & Yellow & 0.301 \\
\hline & ADH -25 & Bangarpet & Yellow & 0.208 \\
\hline & ADH -26 & Malur & Orange & 0.590 \\
\hline & ADH -27 & Bangarpet & Light yellow & 0.725 \\
\hline & ADH -28 & Kolar & Yellow & 0.255 \\
\hline
\end{tabular}

dominant pollen $(>45 \%)$, secondary pollen $(16-45 \%)$, important minor pollen (3-15\%) and minor pollen (<3\%) by constructing pollen spectrum (Louveaux et al., 1978; White, 2005). Furthermore, the pollen morphology of the identified pollen from the honey samples was also documented.

\section{RESULTS AND DISCUSSION}

Kolar district has established beekeeping centres, maintained by the Department of Industries and Commerce of Apiculture wing. The present study identified the bee foraging plants of apiculture importance with particular reference to floral fidelity. The giant bee, $A$. dorsata is a widely distributed wild bee and is one of the important natural pollinators with high foraging potential (Neupane et al., 2006). Twenty-eight honey samples collected from 5 different regions in the study area were subjected to Melittopalynological studies to identify its honey plant resources. The colour, Optic density and place of the collection are documented in Table 1. The colour of the collected honey samples varied from light yellow to dark red. This variation may be due to floral source or exposure of honey to high temperature (Matos and Santos, 2016). The colour classification of honey is very important for commercial purpose. The optical density of the honey samples ranged from 0.208-0.996. Melittopalynological analysis is one of the main tools to determine the honey's botanical origin and differentiate the type of honey as unifloral or multifloral (Rodopoulou et al., 2018). A wide variety of pollen types representing their plant sources and their frequency classes were recognized in each honey samples and listed in Table 2. Among 28 honey samples analysed, 10 samples were identified as multifloral, whereas 18 were unifloral with predominant pollen types such as S. cumini, P. pinnata, Eucalyptus sp, G. abyssinica, $P$. guajava and $C$. sativum. Each count was found to be above $45 \%$ (Table 2).

The pollen type of Eucalyptus sp. was identified in 8 honey samples collected from the study area. The two different species, $E$. globules and $E$. citriodora have been known to occur in this region, has a significant role in providing nectar and pollen to $A$. dorsata. Bees 
Table 2. Pollen spectrum of honey samples collected from Kolar District.

\begin{tabular}{|c|c|c|c|c|c|}
\hline $\begin{array}{l}\text { Honey } \\
\text { sample }\end{array}$ & $\begin{array}{l}\text { Predominant } \\
\text { pollen types } \\
45 \% \text { \& above }\end{array}$ & $\begin{array}{l}\text { Secondary } \\
\text { pollen types } \\
(16-45 \%) \\
\end{array}$ & $\begin{array}{l}\text { Important minor } \\
\text { pollen types } \\
(3-15 \%) \\
\end{array}$ & $\begin{array}{l}\text { Minor pollen types } \\
\text { (Less than } 3 \% \text { ) }\end{array}$ & $\begin{array}{l}\text { Honey } \\
\text { types }\end{array}$ \\
\hline $\mathrm{ADH}-1$ & S. cumini & $\begin{array}{l}\text { Eucalyptus } \\
\text { C. indicum }\end{array}$ & $\begin{array}{l}\text { C. eqisetofolia, } \\
\text { C. sativum, } \\
\text { C. nucifera }\end{array}$ & $\begin{array}{l}\text { D. regia, M. pudica, Poaceae, } \\
\text { H. auriculata, Amaranthaceae, } \\
\text { Fabaceae, C. bonplandianum, } \\
\text { C. lemon, }\end{array}$ & Unifloral \\
\hline $\mathrm{ADH}-2$ & P. pinnata & -------- & $\begin{array}{l}\text { Eucalyptus, } A \text {. } \\
\text { catechu, A. lebbeck }\end{array}$ & $\begin{array}{l}\text { M. pudica, C. nucifera, } P \text {. ptero- } \\
\text { carpum, Poaceae, E. alsinoides }\end{array}$ & Unifloral \\
\hline ADH-3 & ------- & P. guajava & $\begin{array}{l}\text { P. Pterocarpum, } \\
\text { C. nucifera }\end{array}$ & $\begin{array}{l}\text { M. pudica, Asteraceae, D. met- } \\
\text { al, E. alsinoides, A. catechu, } \\
\text { Poaceae, C. sativum. }\end{array}$ & Multifloral \\
\hline $\mathrm{ADH}-4$ & Eucalyptus & ------- & $\begin{array}{l}\text { Asteraceae, M. pudi- } \\
\text { ca, A. indica }\end{array}$ & $\begin{array}{l}\text { C. nucifera, S. jambosa, Ama- } \\
\text { ranthaceae sp., Cassia sp., O. } \\
\text { sanctum, C. lemon. }\end{array}$ & Unifloral \\
\hline ADH-5 & G. abyssinica & Eucalyptus & $\begin{array}{l}\text { H. auriculata, } \\
\text { M. pudica, C. } \\
\text { nurifera, O. sanctum, } \\
\text { C. benghalensis, }\end{array}$ & $\begin{array}{l}P . \text { hysterophorus, Poaceae, } B \text {. } \\
\text { nigra, Fabaceae, J. simplex, } T \text {. } \\
\text { angustata, O. sanctum }\end{array}$ & Unifloral \\
\hline ADH-6 & P. guajava & C. nucifera & $\begin{array}{l}\text { Asteraceae, } D . \\
\text { metal, C. lemon. }\end{array}$ & $\begin{array}{l}\text { C. argentea, Poaceae, T. an- } \\
\text { gustata, Fabaceae, A. catechu, } \\
\text { C. equisetifolia, O. sanctum, C. } \\
\text { bonplandianum, A. chundra. }\end{array}$ & Unifloral \\
\hline ADH-7 & Eucalyptus & ------- & $\begin{array}{l}\text { M. pudica, B. nigra, } \\
\text { Asteraceae, } \\
\text { C. nucifera, } \\
\text { C. benghalensis, }\end{array}$ & $\begin{array}{l}\text { H. auriculata, M. alba, O. sanc- } \\
\text { tum, Poaceae, C. sativum. }\end{array}$ & Unifloral \\
\hline ADH-8 & ----- & $\begin{array}{l}\text { Asteraceae, } \\
\text { Eucalyptus }\end{array}$ & $\begin{array}{l}\text { C. nucifera, } \\
\text { M. pudica, } \\
\text { Amaranthus sp. }\end{array}$ & $\begin{array}{l}\text { A. catechu, Poaceae, H. auricu- } \\
\text { lata, C. benghalensis, R. indica. }\end{array}$ & Multifloral \\
\hline ADH-9 & Eucalyptus & M. pudica & $\begin{array}{l}\text { Asteraceae, } \\
\text { C. nucifera, } \\
\text { O. sanctum. }\end{array}$ & $\begin{array}{l}\text { B. nigra, } A \text {. catechu, C. ben- } \\
\text { ghalensis, } H \text {. auriculata, C. } \\
\text { lemon, Solanaceae, R. com- } \\
\text { munis. }\end{array}$ & Unifloral \\
\hline ADH-10 & Eucalyptus & M. pudica & $\begin{array}{l}\text { Asteraceae, } \\
\text { C. nucifera, } \\
\text { O. sanctum. }\end{array}$ & $\begin{array}{l}\text { A. catechu, Fabaceae, B. nigra, } \\
\text { Poaceae, C. benghalensis. }\end{array}$ & Multifloral \\
\hline $\mathrm{ADH}-11$ & Eucalyptus & -------- & $\begin{array}{l}\text { Asteraceae, } \\
\text { M. pudica, } \\
\text { O. sanctum }\end{array}$ & $\begin{array}{l}\text { Amaranthus sp., H. auriculata, } \\
\text { C. sativum, C. nucifera, D. met- } \\
\text { al, T. terrestris }\end{array}$ & Unifloral \\
\hline ADH-12 & Eucalyptus & ------- & $\begin{array}{l}\text { C. sativum, } \\
\text { C. nucifera, } \\
\text { H. auriculata, }\end{array}$ & $\begin{array}{l}\text { M. pudica, Solanaceae, P. syl- } \\
\text { vestris, C. lemon, O. sanctum. }\end{array}$ & Unifloral \\
\hline $\mathrm{ADH}-13$ & Eucalyptus & H. auriculate & $\begin{array}{l}\text { Asteraceae, } \\
\text { C. sativum, } \\
\text { C. nucifera. }\end{array}$ & $\begin{array}{l}\text { Amaranthus sp., O. sanctum, } B \text {. } \\
\text { nigra, M. pudica. }\end{array}$ & Unifloral \\
\hline $\mathrm{ADH}-14$ & ------ & $\begin{array}{l}\text { Eucalyptus, } \\
\text { Bignoniaceae }\end{array}$ & $\begin{array}{l}\text { Asteraceae, } \\
\text { C. sativum, } \\
\text { G. sepium. }\end{array}$ & $\begin{array}{l}\text { P. sylvestris, Poaceae, Aster- } \\
\text { aceae, C. nucifera, Citrus sp., } \\
\text { M. pudica, Solanaceae, Acacia } \\
\text { sp. }\end{array}$ & Multifloral \\
\hline ADH-15 & ------ & $\begin{array}{l}\text { Asteraceae, } \\
\text { Eucalyptus }\end{array}$ & $\begin{array}{l}\text { C. sativum, } \\
\text { O. sanctum, } \\
\text { H. auriculata, } M \text {. } \\
\text { pudica, C. nucifera. }\end{array}$ & $\begin{array}{l}\text { T. argentia, P. sylvestris, Faba- } \\
\text { ceae, Acacia sp. Amaranthus } \\
\text { sp. C. bonplandianum, D. met- } \\
\text { al. Poaceae, }\end{array}$ & Multifloral \\
\hline ADH-16 & ----- & $\begin{array}{l}\text { Eucalyptus, } \\
\text { Bignoniaceae }\end{array}$ & $\begin{array}{l}\text { Asteraceae, } \\
\text { C. nucifera }\end{array}$ & $\begin{array}{l}\text { A. catechu, Acacia sp., Poace- } \\
\text { ae, M. pudica, R. communis, C. } \\
\text { sativum, A. lebbeck. }\end{array}$ & Multifloral \\
\hline $\mathrm{ADH}-17$ & P. guajava & ------ & $\begin{array}{l}\text { P. pinnata, } \\
\text { Asteraceae, } \\
\text { Eucalyptus. }\end{array}$ & $\begin{array}{l}\text { C. nucifera, Citrus sp., A. indi- } \\
\text { ca, Poaceae, C. bonplandi- } \\
\text { anum, P. sylvestris, D. regia, }\end{array}$ & Unifloral \\
\hline ADH-18 & -------- & $\begin{array}{l}\text { Eucalyptus, } \\
\text { Syzygium sp., } \\
\text { M. pudica }\end{array}$ & $\begin{array}{l}\text { C. nucifera, } \\
\text { Asteraceae }\end{array}$ & $\begin{array}{l}P . \text { pinnata, Poaceae, } P \text {. guaja- } \\
\text { va, } P \text {. sylvestris, Fabaceae, C. } \\
\text { bonplandianum. }\end{array}$ & Multifloral \\
\hline
\end{tabular}


Table 2. Contd.......

\begin{tabular}{|c|c|c|c|c|c|}
\hline ADH-19 & Eucalyptus & $\begin{array}{l}\text { Syzygium sp., } \\
\text { Asteraceae }\end{array}$ & $\begin{array}{l}\text { P. guajava, M. pudi- } \\
\text { ca, Commelina sp., } \\
\text { C. nucifera. }\end{array}$ & $\begin{array}{l}\text { H. auriculata, Amaranthus sp., } \\
\text { C. sativum, Poaceae, O. sanc- } \\
\text { tum, V. negundo }\end{array}$ & Unifloral \\
\hline ADH-20 & ------- & $\begin{array}{l}\text { Eucalyptus, M. } \\
\text { pudica }\end{array}$ & $\begin{array}{l}\text { Asteraceae, C. sa- } \\
\text { tivum, S. laurifolius }\end{array}$ & $\begin{array}{l}\text { O. sanctum, Commelina sp. C. } \\
\text { lemon, Poaceae, H. auriculata, } \\
\text { Z. oenopilia }\end{array}$ & Multifloral \\
\hline ADH-21 & P. guajava & M. pudica & $\begin{array}{l}\text { Asteraceae, Syzygi- } \\
\text { um sp., Eucalyptus, } \\
\text { Ipomea sp. }\end{array}$ & $\begin{array}{l}\text { C. equisetifolia, C. nucifera, } \\
\text { Amaranthus sp. B. nigra, Citrus } \\
\text { sp., Commelina sp., C. sativum. }\end{array}$ & Unifloral \\
\hline ADH-22 & P. guajava & $\begin{array}{l}\text { Asteraceae, } M \text {. } \\
\text { pudica }\end{array}$ & $\begin{array}{l}\text { Syzygium sp., C. } \\
\text { nucifera, Eucalyptus, } \\
\text { O. sanctum. }\end{array}$ & $\begin{array}{l}\text { C. benghalensis, C. argentea, } \\
\text { B. nigra, Solanaceae, C. lemon, } \\
\text { H. auriculata, Cyperus sp., Po- } \\
\text { aceae. }\end{array}$ & Unifloral \\
\hline ADH-23 & Eucalyptus & $\begin{array}{l}\text { Asteraceae, M. } \\
\text { pudica }\end{array}$ & $\begin{array}{l}\text { Amaranthus sp., C. } \\
\text { sativum, B. nigra }\end{array}$ & $\begin{array}{l}\text { C. nucifera, Acacia sp., C. bon- } \\
\text { plandianum, O. sanctum, H. } \\
\text { auriculata, T. asiatica. }\end{array}$ & Unifloral \\
\hline ADH-24 & Eucalyptus & $\begin{array}{l}\text { C. nucifera, } T \text {. } \\
\text { indicus }\end{array}$ & $\begin{array}{l}\text { Asteraceae, M. pudi- } \\
\text { ca, J. simplex }\end{array}$ & $\begin{array}{l}\text { Amaranthus sp., Cyperus sp., } \\
\text { Acacia sp., O. sanctum, C. sa- } \\
\text { tivum, H. auriculata, C. inerme, } \\
\text { C. bonplandianum. }\end{array}$ & Unifloral \\
\hline ADH-25 & C. sativum & O. sanctum & $\begin{array}{l}\text { Eucaluptus, Aster- } \\
\text { aceae }\end{array}$ & $\begin{array}{l}\text { C. nucifera, C. benghalensis, } \\
\text { Amaranthus sp., Poaceae, } B \text {. } \\
\text { hispida, C. inerme. }\end{array}$ & Unifloral \\
\hline ADH-26 & ------ & $\begin{array}{l}\text { Eucalyptus, C. } \\
\text { nucifera }\end{array}$ & $\begin{array}{l}\text { Asteraceae, M. pudi- } \\
\text { ca, C. sativum, M. } \\
\text { indica }\end{array}$ & $\begin{array}{l}\text { A. catechu, Amaranthus sp., } H \text {. } \\
\text { auriculata, Poaceae, O. sanc- } \\
\text { tum, A. marmelos }\end{array}$ & Multifloral \\
\hline ADH-27 & Syzygium sp. & M. pudica & $\begin{array}{l}\text { Eucalyptus, C. nucif- } \\
\text { era, } H \text {. auriculalta }\end{array}$ & $\begin{array}{l}\text { O. sanctum, C. sativum, Citrus } \\
\text { sp., } H \text {. annus }\end{array}$ & Unifloral \\
\hline ADH-28 & P. pinnata & $\begin{array}{l}\text { Eucalyptus, } \\
\text { Asteraceae }\end{array}$ & $\begin{array}{l}\text { F. elephantum, E. } \\
\text { alsinoides }\end{array}$ & $\begin{array}{l}\text { G. robusta, Rubiaceae, } D \text {. } \\
\text { regia, Poaceae, } A \text {. chundra }\end{array}$ & Unifloral \\
\hline
\end{tabular}

prefer pollen and nectar of Eucalyptus tree to a larger extent, because of the tree flowers throughout the year (Tamar Keasar and Avi Shmida, 2009; Layek and Karmakar, 2018). This is evident in the honey sample of $A$. dorsata honey (ADH-4, 7, 9,12, 13, 19, 23 and 24) and all these honey samples were unifloral type. The other pollen types identified with Eucalyptus were as Acacia sp., B. nigra, Cassia sp., C. lemon, Clerodendrum inerme, Cocos nucifera, Commelina benghalensis, Coriandrum sativum, Croton bonplandianum, Cyperus sp., Hygrophila auriculata, Mimosa pudica, Morus alba, Ocimum sanctum, Phoenix sylvestris, Ricinus communi and $S$. jambosa. Likewise, Psidium guajava pollen was identified as dominant in 4 honey samples collected from Mulbagal (ADH-6), Malur (ADH-21), Bangarpet (ADH-21) and Kolar (ADH-24). $P$. guajava is a small tree, belongs to the Myrtaceae family that flowers throughout the year. Psidium flowers produce a sufficient amount of pollen and nectar to honey bees (Anita et al., 2012). The pollen spectrum of this unifloral honey also showed the pollen types of $P$. pinnata, Eucalyptus Sp. C. nucifera, Azaadirachta indica, C. lemon, C. bonplandianum, P. sylvestris, Delonix regia, Datura metal, Celosia argentea, Typha angustata, Casuarina equisetifolia, Brassica nigra, C. sativum, C. benghalensis, Hygrophila auriculata, Amaranthus sp. and Cyperus sp.
S. cumini (ADH-1 and 27) and $P$. pinnata (ADH-2 and 28) pollen type were found in 2 samples each. $S$. cumini is a large evergreen tree found all along the avenues and around the forest area. It is an important source of nectar and pollen for honeybees because the tree flowers from February and continues to bloom till the end of April (Abou-Shaara, 2014). Eucalyptus, C. eqisetofolia, C. sativum, C. nucifera, D. regia, M. pudica, $H$. auriculata, C. lemon, C. bonplandianum and $O$. sanctum pollen types were associated with this unifloral honey. Whereas $P$. pinnata, a much branched bushy tree, widely cultivated medicinal application. A nontimber tree flowers profusely during the onset of summer month, it closes before the early monsoon. The Pongamia honey associated with Eucalyptus sp., $A$. catechu, M. pudica, C. nucifera, Peltophorum pterocarpum, Evolvulus alsinoides, Ferronia elephantum, Grevillea robusta and Delonix regia. Further, pollen spectrum revealed the association of Guizotia abyssinica uniforal honey sample (ADH-5) was Eucalyptus Sp., Hygrophila auriculata, M. pudica, Commelina benghalensis, C. nurifera, O. sanctum, P. hysterophorus, $B$. nigra, Justicia simplex and Typha angustata. Guizotia abyssinica is an annual erect herb to a height of 3 feet. It is cultivated as a minor crop along with ragi or groundnut which flowers during August to December. Coriander pollen occurs as dominant pollen in the sam- 
Table 3. Floral calendar of bee forage plants of Kolar district during 2017-2019.

\begin{tabular}{|c|c|c|c|c|c|}
\hline $\begin{array}{l}\text { SI. } \\
\text { No. }\end{array}$ & Plant species & Family & Habit & Flowering period & $\begin{array}{l}\text { Economic } \\
\text { importance }\end{array}$ \\
\hline 1 & Acacia catechu (L.f.) Willd. & Fabaceae & Tree & June-October & Medicinal \\
\hline 2 & Acacia chundra (Roxb. ex Rottler) & Fabaceae & Tree & April-August & Medicinal \\
\hline 3 & Albizia lebbeck (L.) Willd & Fabaceae & Tree & July-November & Timber \\
\hline 4 & Amaranthus spinosus L. & Amaranthaceae & Herb & July-October & Vegetable \\
\hline 5 & Areca catechu L. & Arecaceae & Tree & January-March & Nut \\
\hline 6 & Azadirachta indica Juss. & Meliaceae & Tree & March-April & Medicinal \\
\hline 7 & Bauhinia purpurea L. & Fabaceae & Tree & August-November & Ornamental \\
\hline 8 & Borreria hispida (L.) Schum. & Rubiaceae & Herb & January-December & Medicinal \\
\hline 9 & Borreria stricta (L.f.) Schum. & Rubiaceae & Herb & January-December & Medicinal \\
\hline 10 & Brassica nigra (L.) Koch. & Brasicacece & Herb & March-June & Oil Yielding \\
\hline 11 & Caesalpina pulcherrima (L.) Swart. & Fabaceae & Tree & January-December & Ornamental \\
\hline 12 & Cassia mimosoides $\mathrm{L}$. & Fabaceae & Tree & August-October & Ornamental \\
\hline 13 & $\begin{array}{l}\text { Casuarina equisetifolia J.R.and } \\
\text { G.forsst. }\end{array}$ & Casuarinaceae & Tree & February-September & Timber \\
\hline 14 & Celosia argentea L. & Amaranthaceae & Herb & June-September & Vegetable \\
\hline 15 & Chrysanthemum indicum L. & Asteracece & Herb & January-December & Ornamental \\
\hline 16 & Citrus aurantium L. & Rutaceae & Tree & April-May & Fruit \\
\hline 17 & Citrus medica L & Rutaceae & Tree & April-May & Fruit \\
\hline 18 & Clerodendrum inerme & Lamiaceae & Shrub & October-March & Medicinal \\
\hline 19 & Cocos nucifera L. & Arecaceae & Tree & January-December & Oil Yielding \\
\hline 20 & Commelina benghalensis L. & Commelinaceae & Shrub & June-October & Medicinal \\
\hline 21 & Coriandrum sativum $\mathrm{L}$. & Apiaceae & Herb & June-July & Vegetable \\
\hline 22 & Croton bonplandianum Baill. & Fabaceae & Shrub & January-December & Medicinal \\
\hline 23 & Cyperus Sp & Cyperacece & Herb & May-June & Medicinal \\
\hline 24 & Datura metal L. & Solanaceae & Shrub & January-June & Weed \\
\hline 25 & Delonix regia (Boj.ex.) Raf. & Fabaceae & Tree & June-July & Ornamental \\
\hline 26 & Eucalyptus Sp. & Myrtaceae & Tree & March-June & Timber \\
\hline 27 & Evolvulus alsinoides L. & Convolvulaceae & Shrub & February-June & Medicinal \\
\hline 28 & Feronia elephanum Correa & Rutaceae & Shrub & January-December & Fruit \\
\hline 29 & $\begin{array}{l}\text { Gliricidia sepium (Jacq) Kunth ex. } \\
\text { Steud. }\end{array}$ & Fabaceae & Tree & November-March & Medicinal \\
\hline 30 & Grevillea robust A. Cunn & Proteaceae & Tree & January-December & Timber \\
\hline 31 & Guizotia abyssinica Cass. & Asteraceae & Herb & May-October & Oil yielding \\
\hline 32 & Helianthus annus L. & Asteraceae & Shrub & July-October & Oil Yielding \\
\hline 33 & $\begin{array}{l}\text { Hygrophila auriculata (Schum) } \\
\text { Heine. }\end{array}$ & Acanthaceae & Herb & October-April & Medicinal \\
\hline 34 & Ipomoea sp. L & Convolvulaceae & Herb & January-December & Weed \\
\hline 35 & Justicia simplex Don. & Acanthaceae & Herb & October-December & Weed \\
\hline 36 & Mangifera indica L. & Anacardiaceae & Tree & March-April & Fruit \\
\hline 37 & Mimosa pudica L. & Fabaceae & Herb & Januaru-December & Weed \\
\hline 38 & Morus alba L. & Moraceae & Tree & May-June & Silk Production \\
\hline 39 & Ocimum sanctum $\mathrm{L}$. & Lamiaceae & Herb & January-December & Medicinal \\
\hline 40 & Partheniumhyserophorus L. & Asteraceae & Herb & January-December & Weed \\
\hline 41 & $\begin{array}{l}\text { Peltophorum pterocarpum (DC) } \\
\text { Baker. }\end{array}$ & Fabaceae & Tree & March-April & Ornamental \\
\hline 42 & Phoenix sylvestris (L.) Roxb. & Arecaceae & Tree & June-August & Fruit \\
\hline 43 & Pongamia pinnata Vent. & Fabaceae & Tree & April-June & Oil Yielding \\
\hline 44 & Prosopis julifera D.C. & Fabaceae & Tree & August-Feb & Medicinal \\
\hline 45 & Psidium guajava L. & Myrtaceae & Tree & February-April & Fruit \\
\hline 46 & Ricinus communis $\mathrm{L}$. & Euphorbiaceae & Tree & March-April & Oil Yielding \\
\hline 47 & Rosa indica L. & Rosaceae & Shrub & \multirow{4}{*}{$\begin{array}{l}\text { January-December } \\
\text { February-April } \\
\text { March-April } \\
\text { April-May, August- } \\
\text { September }\end{array}$} & Ornamental \\
\hline 48 & Sapindus laurifolius Vahl. & Sapindaceae & Tree & & Medicinal \\
\hline 49 & Syzygium cumini (L.) Skeels. & Myrtaceae & Tree & & Fruit \\
\hline 50 & Tabebuia argentia (R \& S) Britt. & Bignoniaceae & Tree & & Ornamental \\
\hline $\begin{array}{l}51 \\
52\end{array}$ & $\begin{array}{l}\text { Tamarindus indicus L. } \\
\text { Toddalia asiatica (L.) Lamk. }\end{array}$ & $\begin{array}{l}\text { Fabaceae } \\
\text { Rutaceae }\end{array}$ & $\begin{array}{l}\text { Tree } \\
\text { Herb }\end{array}$ & $\begin{array}{l}\text { March-May } \\
\text { March-September }\end{array}$ & $\begin{array}{l}\text { Fruit } \\
\text { Medicinal }\end{array}$ \\
\hline 53 & Tribulus terrestris $\mathrm{L}$. & Zygophyllaceae & Herb & March-May & Weed \\
\hline 54 & Typha angustata L. & Typhaceae & Herb & May-July & Medicinal \\
\hline 55 & Vitex negundo Mill. & Lamiaceae & Shrub & July-August & Medicinal \\
\hline 56 & Ziziphus oenoplia (L.) Mill. & Rhamnaceae & Tree & July-November & Fruit \\
\hline
\end{tabular}




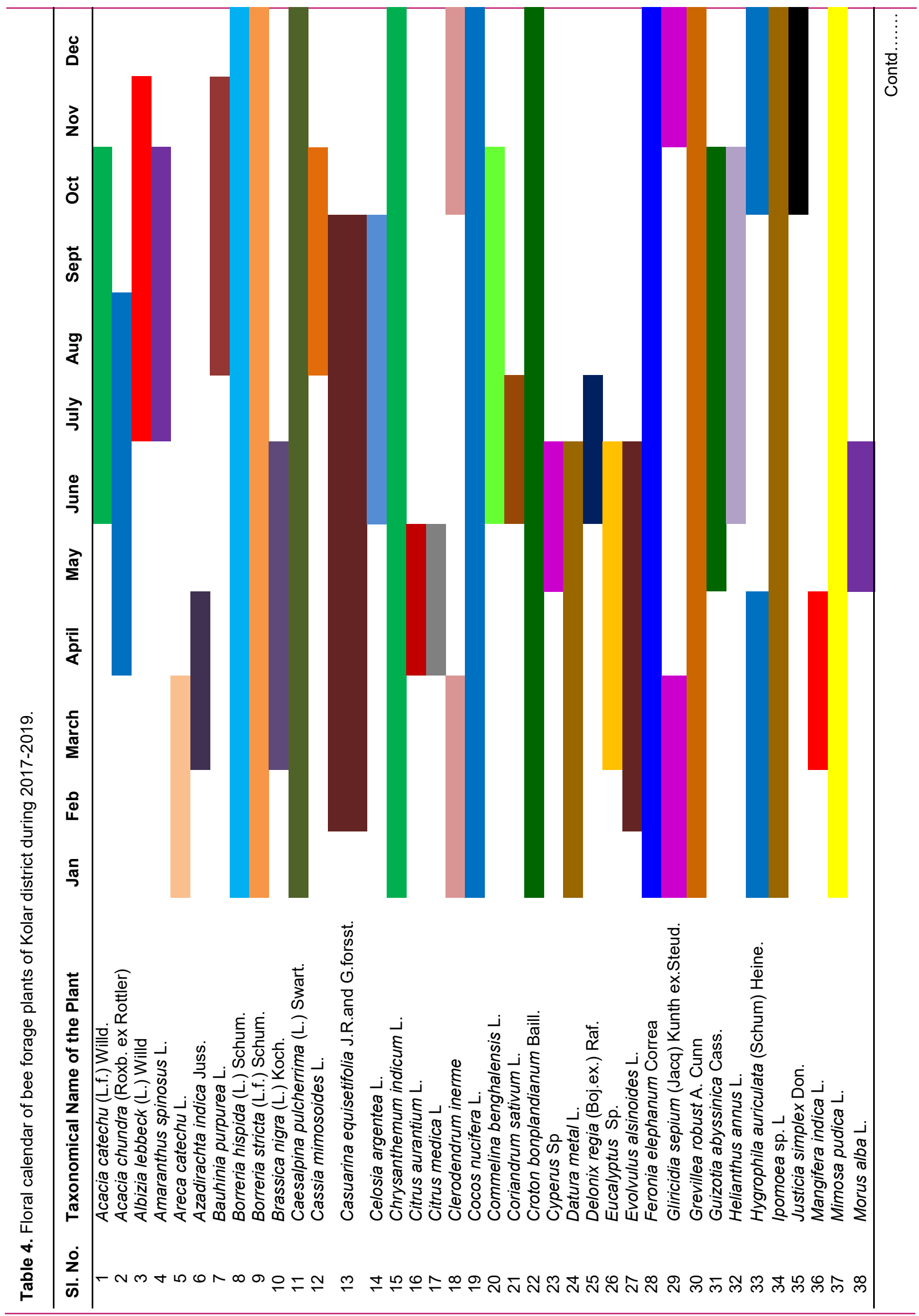



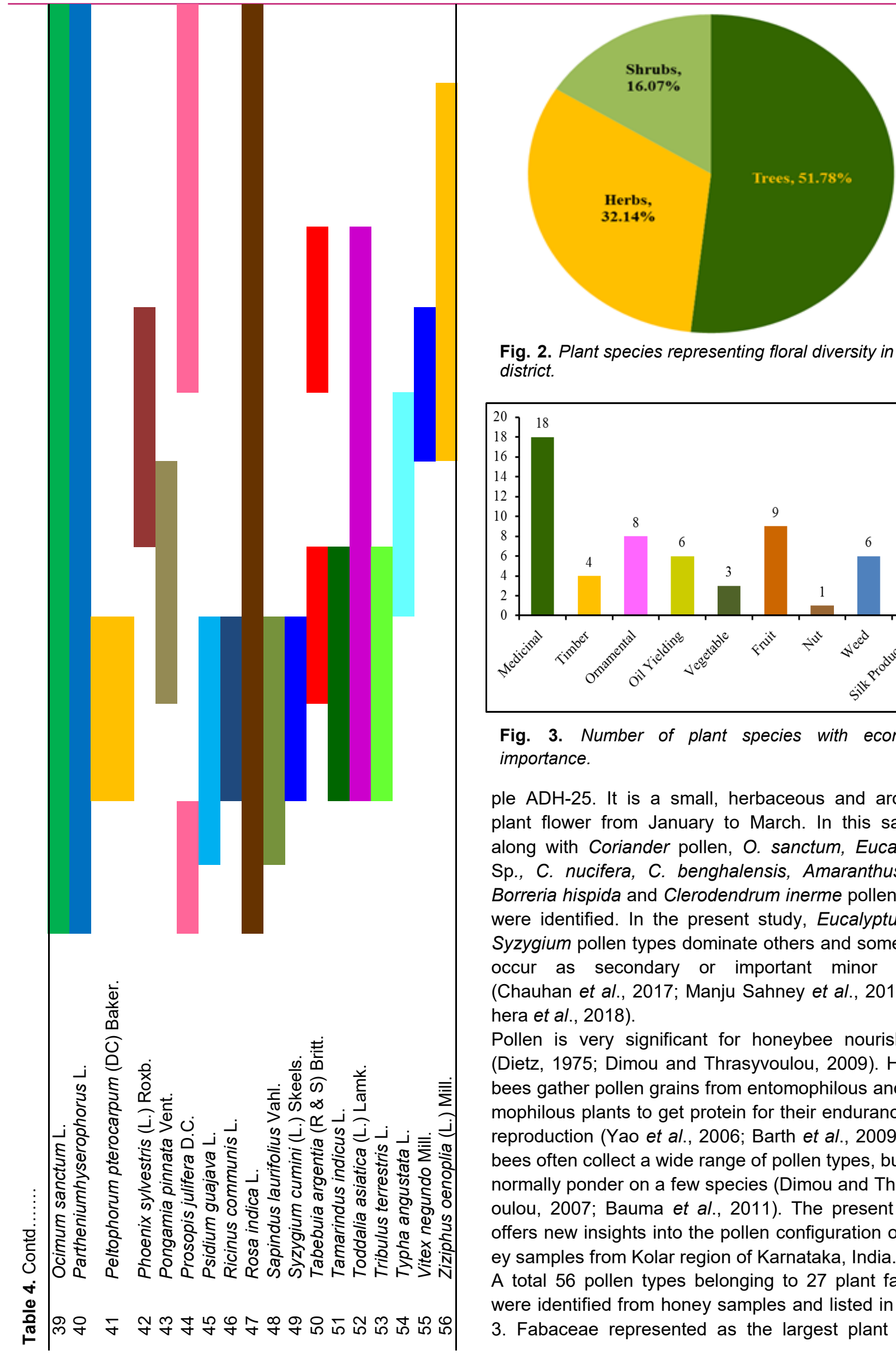

Fig. 2. Plant species representing floral diversity in Kolar district.

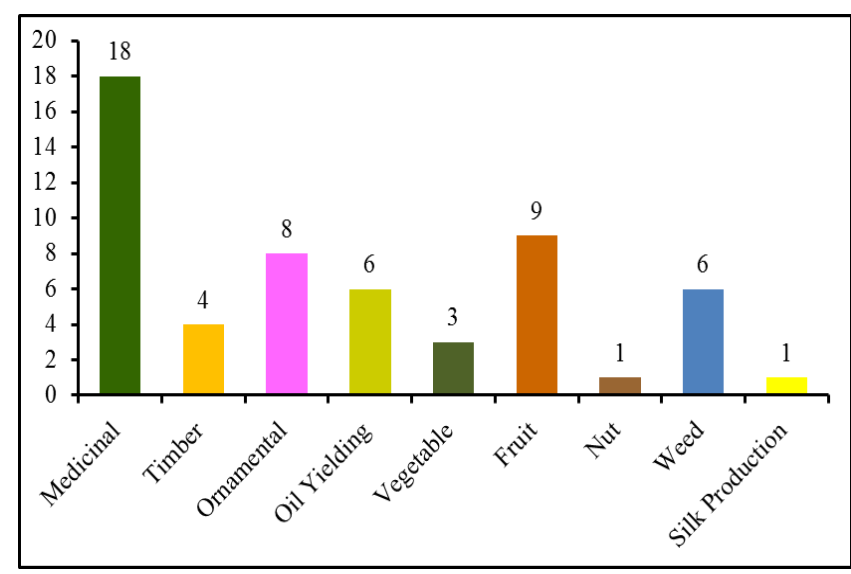

Fig. 3. Number of plant species with economic importance.

ple $A D H-25$. It is a small, herbaceous and aromatic plant flower from January to March. In this sample, along with Coriander pollen, O. sanctum, Eucaluptus Sp., C. nucifera, C. benghalensis, Amaranthus Sp., Borreria hispida and Clerodendrum inerme pollen types were identified. In the present study, Eucalyptus and Syzygium pollen types dominate others and sometimes occur as secondary or important minor pollen (Chauhan et al., 2017; Manju Sahney et al., 2018; Behera et al., 2018).

Pollen is very significant for honeybee nourishment (Dietz, 1975; Dimou and Thrasyvoulou, 2009). Honeybees gather pollen grains from entomophilous and anemophilous plants to get protein for their endurance and reproduction (Yao et al., 2006; Barth et al., 2009). The bees often collect a wide range of pollen types, but they normally ponder on a few species (Dimou and Thrasyvoulou, 2007; Bauma et al., 2011). The present study offers new insights into the pollen configuration of honey samples from Kolar region of Karnataka, India.

A total 56 pollen types belonging to 27 plant families were identified from honey samples and listed in Table 3. Fabaceae represented as the largest plant family 
Table 5. Pollen morphology of some dominant pollen types of the study area.

\begin{tabular}{|c|c|c|c|c|c|}
\hline $\begin{array}{l}\text { SI. } \\
\text { No. }\end{array}$ & Taxon & Aperture & Ornamentation & $\begin{array}{l}\text { Size, Shape \& Exine } \\
\text { pattern }\end{array}$ & Photomicrograph \\
\hline 1 & Acacia sp. & Tricolpoid & Striato Reticulate & $\begin{array}{l}38-45,50-58 \mu \mathrm{m} \\
\text { exine } 1.5-3 \mu \mathrm{m}\end{array}$ & \\
\hline 2 & Asteraceae & Tricolporate & Echinulate & $35-38 \mu \mathrm{m}$, exine $5 \mu \mathrm{m}$ & \\
\hline 3 & A. indica & Tetracolporate & Reticulate & $\begin{array}{l}\text { Prolate spheroidial, } \\
\text { exine } 1-0.5 \mu \mathrm{m}\end{array}$ & \\
\hline 4 & B. purpurea. & Tricolporate & Striate & $\begin{array}{l}25 \times 30 \mu \mathrm{m} \text {, exine } 1.5 \\
\mu \mathrm{m}\end{array}$ & \\
\hline 5 & B. nigra & Tricolpate & Reticulate & $\begin{array}{l}28-31 \mu \mathrm{m} \text { subspheri- } \\
\text { cal, exinie } 1.5 \mu \mathrm{m}\end{array}$ & \\
\hline 6 & Citrus sp. & Tetracolporate & Reticulate & $\begin{array}{l}30-34,24-31 \mu \mathrm{m} \\
\text { amb tetragonal }\end{array}$ & \\
\hline 7 & C. nucifera & Monosulcate & Granulate & Oblate & \\
\hline 8 & C. sativum & $\begin{array}{l}\text { Tricolporate ora } \\
\text { elliptic }\end{array}$ & Granulate & $\begin{array}{l}25-28,40-50 \mu \mathrm{m}, \\
\text { exine } 2 \mu \mathrm{m}\end{array}$ & \\
\hline
\end{tabular}


Table 5. Contd.......

9

C. bonplandianum.

Omni aperturate

Reticulate with crotonoid pattern

$46-48 \mu \mathrm{m}$, exine $3 \mu \mathrm{m}$

36-41,

26-

Granulate

$34 \mu \mathrm{m}$, exine $1 \mu \mathrm{m}$

$50 \times 50 \mu \mathrm{m}$ spheroidal, exine $4 \mu \mathrm{m}$

19-22x26-31 $\mu \mathrm{m}$ exine $1 \mu \mathrm{m}$ amb semiangular exine $0.5 \mu \mathrm{m}$

25-28, 29$31 \mu \mathrm{m}$, exine $1.5 \mu \mathrm{m}$

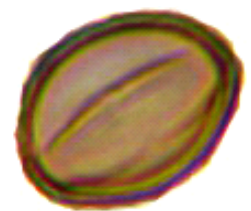

25-30 $\mu \mathrm{m}$ spheroidal
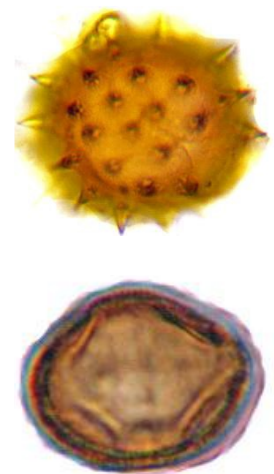

Spheroidal, exine $1 \mu \mathrm{m}$

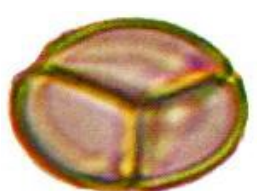

$55-75 \mu \mathrm{m}$ suboblate exine $4 \mu \mathrm{m}$

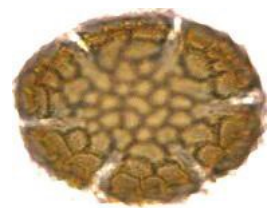


Table 5. Contd.......

18

P. pterocarpum Tricolporate

Reticulate

19

P. pinnata

Tricolporate

Psilate

20

P. guajava

Tricolporate

Psilate

21

R. communis

Tricolporate

Reticulate

22

R. indica

Tricolporate

Striate reticulate

$25 \times 45 \mu \mathrm{m}$ exine $2.5 \mu \mathrm{m}$

$12 \times 28 \mu \mathrm{m}$ oblate exine $1.5 \mu \mathrm{m}$

44-31 $\mu \mathrm{m}$ subprolate to prolate

35-35 $\mu \mathrm{m}, \quad \mathrm{amb}$ circular, exine $2 \mu \mathrm{m}$
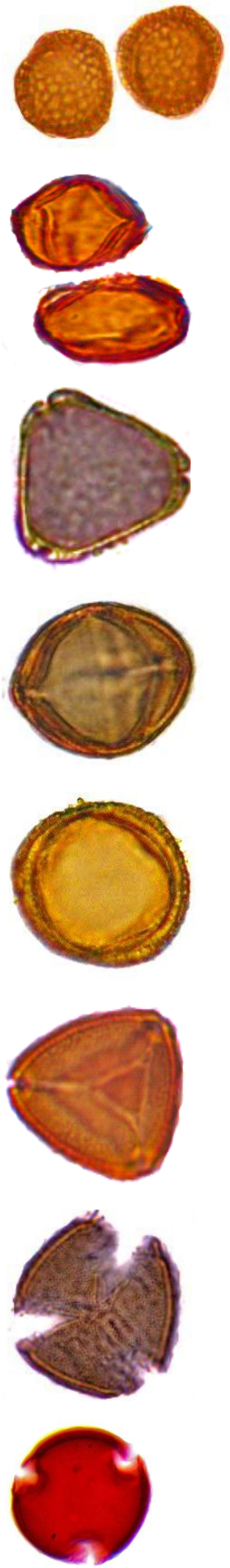

with 14 species contributing to the honey production in the study area. In addition, Rutaceae and Asteraceae comprised of 4 species each, followed by Arecaceae, Lamiaceae and Myrtaceae recorded with 3 species each. Convolvulaceae, Amaranthaceae, Rubiaceae and Acanthaceae were found with 2 species each and Anacardiaceae, Apiaceae, Bignoniaceae, Brasicaceae, Casurinaceae, Commelinaceae, Cyperaceae, Euphor- biaceae, Moraceae, Proteaceae comprised of single species each.

All the 56 pollen types recorded comprised several plant types such as trees, shrubs and herbs. Among the reported habit, there was a dominance of the tree with $51.78 \%$, followed by herbs and shrubs with $32.14 \%$ and $16.07 \%$, respectively, represent floral diversity (Fig. 2). The economic importance of the identi- 
fied plants of apiculture importance was categorized as medicinal, ornamental, vegetable, timber, oil yielding, weeds, fruits and nuts (Fig. 3). According to the present result, predominant and secondary dominant pollen types with medicinal importance were A. catechu, $A$. chundra, A. indica, Gliricidia sepium, C. inerme, B. hispida, B. stricta, C. benghalensis, Croton bonplandianum, Cyperus sp., Evolvulus alsinoides, $H$. auriculata, O. sanctum, Prosopis julifera, Sapindus laurifolius, Toddalia asiatica, Typha angustata and Vitex negundo. Pollen types of Bauhinia purpurea, Caesalpina pulcherrima, Delonix regia, C. mimosoides, Chrysanthemum indicum, Rosa indica, Tabebuia argentia and P. pterocarpum were grouped as ornamental plants. Amaranthus spinosu, Celosia argentea and C. sativum were vegetables and Albizia lebbeck, Casuarina equisetifolia, Eucalyptus sp. and Grevillea robust were timber plants. B. nigra, Guizotia abyssinica, H. annus, C. nucifera, $P$. pinnata and $R$. communis are important oil yielding plants. The fruit and nuts yielding plants were Feronia elephanum, $P$. sylvestris, $P$. guajava, $S$. cumini, C. aurantium, C. medica, Tamarindus indicus, Ziziphus oenoplia and $A$. catechu. Weeds of forage importance to $A$. dorsata in the study area were $D$. metal, M. pudica, P. hyserophorus, Ipomoea sp., J. simplex and Tribulus terrestris, whereas $M$. alba was cultivated for production of silk.

From the study it is observed that, most of the plant species such as B. hispida, B. stricta, C. pulcherrima, C. nucifera, C. indicum, C. bonplandianum, Eucalyptus sps. F. elephanum, G. robust, Ipomoea sp, M. pudica, O. sanctum, P. hyserophorus, $R$. communis and $R$. indica have prolonged blooming period throughout the year. These species provide greater forage potential for honeybees in the study area. Pollen grains are the most important component of honey helps to identify the distribution of floral source of honey. The blooming period of each species was recorded as shown in Table 4. According to the present observation, B. hispida, $B$. stricta, C. pulcherrima, C. indicum, C. nucifera, C. bonplandianum, F. elephanum, G. robust, Ipomoea sp., M. pudica, $O$. sanctum, $P$. hyserophorus and $R$. indica bloom throughout the year. The peak flowering period of most important honey plants were observed during February to June and less blooming period was observed during October to January. Microscopic analysis of honey helps to identify the pollen types with their size, shape and ornamentation. The morphology of pollen grains differ in shapes, exine structure, symmetry and sculpture among plant species. Table 5 demonstrated the morphology of various types of pollen obtained from the present study. The pollen of Acacia sp. are sub globes, whereas $P$. pinnata pollen are spherical. It has been established in this study that the species belongs to the family Asteraceae pollen type were spinolous, Myrtaceae pollen types are colporate and prolate. But there is variability in the pollen type of the species belongs to family Fabaceae. Therefore, it is essential to examine a large number of pollen grains from one family in order to obtain a complete knowledge of different types within that family.

\section{Conclusion}

The present study contributes to the floral resources of A. dorsata honey produced in Kolar district of Karnataka state. A. dorsata mainly depends on the wild tree and cultivated plant blooms throughout the year as pollen and nectar source. From the results, it is evident that there is a lot of potential in establishing beekeeping industries in the study area. Among 28 honey samples analysed, 18 were unifloral, with the predominant pollen types were C. sativum, E. globulus, G. abyssinica, $S$. cumini, $P$. pinnata and $P$. guajava. The most represented families were Fabaceae, Rutaceae and Asteraceae. These plants may be introduced in social forestry and afforestation programs to enhance the honey yield. Further, the yield of economic crops may also be able to increase by pollination. Assessment of honey bee pollen as a bioindicator of the environment may throw more light on floral diversity.

\section{Conflict of interest}

The author declares that he has no conflict of interest.

\section{REFERENCES}

1. Abou-Shaara, H.F. (2014). The foraging behaviour of honey bees, Apis mellifera: A review. Veterinarni Medicina, 59 (1), 1-10. doi:10.17221/7240-VETMED

2. Agwu, C.O.C. \& Akanbi, T.O. (1985). A palynological study of honey from four vegetation zones in Nigeria. Pollen et Spores, 27, 335-348.

3. Alves, R.F. \& Santos, F.A.R. (2014). Plant sources for bee pollen load production in Sergipe, Northeast Brazil. Palynology, 38(1), 90-100. doi.10.1080/01916122.2013.846 280

4. Anita, M., Sivararam, V. \& Jayaramappa, K.V. (2012) Influence of bee attractants on pollination and yield parameters in guava (Psidium guajava L.). International Journal of Plant Reproductive Biology, 4(1), 41-46.

5. Anklam, E. (1998). A review of the analytical methods to determine the geographical and botanical origin of honey. Food Chemistry, 63, 549-562. doi.org/10.1016/S03088146(98)00057-0

6. Barth, O.M. (1990). Pollen in monofloral honeys from Brazil. Journal of Apicultural Research, 29(2), 89-94. doi.org/10.1080/00218839.1990.11101202

7. Barth, O.M., Munhoz, M.C. \& Luz, C.F.P. (2009). Botanical origin of Apis pollen loads using colour, weight and pollen morphology data. Acta Alimentaria, 38(1), 133-139. doi.org/10.1556/aalim.2008.0026 
8. Baum, K.A., Rubink, W.L., Coulson, R.N. \& Bryant Jr. V.M. (2011). Diurnal patterns of pollen collection by feral honey bee colonies in southern Texas, USA. Palynology, 35(1), 85-93. doi.org/10.1080/01916122.2010.546621

9. Behera, S.S., Ray, R.C. \& Zdolec, N. (2018). Lactobacillus plantarum with functional properties: An approach to increase safety and shelf-life of fermented foods. BioMed Research International, 9361614. doi.org/10.1155/20 18/9361614

10. Chauhan, M.S., Farooqui, A. \& Trivedi, A. (2017). Plants foraged by bees for honey production in northern India: The diverse flora of India and its implications for apiculture. Acta Palaeobotanica, 57(1), 119-132. doi:10.1515/ acpa-2017-0003

11. Deodikar, G.B. \& Thakar, C.V. (1953). A pollen study of honey yielding plants of Mahabaleshwar hills. Apicultural Lab. Bulletin, No.1, 1-6.

12. Deodikar, G.B. (1961). Some aspects of bee botany. Indian Bee Journal, 23, 60-61.

13. Diaz-Losada, E., Ricciardelli-d'Albore, G. \& Saa-Otero, M.P. (1998). The possible use of honeybee pollen loads in characterizing vegetation. Grana, 37(3), 155-163. doi.org/10.1080/00173139809362660

14. Dietz, A. (1975). Nutrition of the adult honey bee. In: The hive and the honey bee, (Graham JM, ed.) Hamilton, Illinois: Dadant \& Sons. pp.125-156.

15. Dimou, M. \& Thrasyvoulou, A. (2007). Seasonal variation in vegetation and pollen collected by honeybees in Thessaloniki, Greece. Grana, 46(4), 292-299. doi.org/10.1080/00173130701760718

16. Dimou, M. \& Thrasyvoulou, A. (2009). Pollen analysis of honeybee rectum as a method to record the bee pollen flora of an area. Apidologie, 40, 124-133. doi.org/10.1051/ apido/2008066

17. Erdtman, G. (1960). The acetolysis method. A revised description. Svensk Botanisk Tidskr, 54, 561-564.

18. Feller-Demalsy, M.J., Parent, J. \& Strachan, A. (1989). Microscopic analysis of honeys from Manitoba, Canada. Journal of Apicultural Research, 28(1), 41-49. doi.org/10.1080/00218839.1989.11100819

19. Garg, A. (2006). Bee botany of Bhimtal in Western Himalayas: Melissopalynological analysis. $1^{\text {st }}$ ed. Dehra Dun: Bishen Singh \& Mahendra Pal Singh.

20. Jesus, M.C., Borges, R.L.B., Souza, B.A., Brandao, H.N. \& Santos, F.A.R. (2015). A study of pollen from light honeys produced in Piaul state, Brazil. Palynology, 39(1), 10124. doi:10.1080/01916122.2014.942440

21. Layek, U. \& Karmakar, P. (2018). Pollen analysis of Apis dorsata Fabricius honeys in Bankura and Paschim Medinipur districts, West Bengal. Grana, 57(4), 298-310. doi:10.1080/00173134.2017.1390604

22. Lieux, M.H. (1980). Acetolysis applied to microscopical honey analysis. Grana, 19, 57-61. doi.org/10.1080/00173138009424988

23. Lin, S.H., Chang, S.Y. \& Chen, S.H. (1993). Nectar and pollen sources for honeybee (Apis cerana Fabr.) in Qinglan mangrove area, Hainan Island, China. Journal of Integrative Plant Biology, 48, 1266-1273.

24. Louveaux, J., Maurizio, A. \& Vorwohl, G. (1978). Methods of melissopalynology. Bee World, 59(4), 139-157. doi.org/10.1080/0005772X.1978.11097714

25. Manju Sahney, Snehlata Rahi, Ajay Kumar \& Roma
Jaiswal. (2018). Melissopalynological studies on winter honeys from Allahabad, Uttar Pradesh, India. Palynology, 42(4), 540-552. doi:10.1080/01916122.2017.1418445

26. Matos, V.R. \& Santos, F.A.R. (2016). Pollen in honey of Melipona scutellaris L. (Hymenoptera: Apidae) in an Atlantic rainforest area in Bahia, Brazil. Palynology, 41, 144156. doi:10.1080/01916122.2015.1115434

27. Maurizio, A. (1975). Microscopy of honey. In: Honey, a comprehensive survey. (Crane E, editor). New York: Crane, Russak and Co., pp. 240-257.

28. Majid, M., Ellulu, M.S. \& Bakar, M.F.A. (2020). Melissopalynological Study, Phenolic Compounds, and Antioxidant Properties, of Heterotrigona itama Honey from Johor, Malaysia. Hindawi Scientifica, Volume 2020, Article ID: 2529592, 9 pages doi.10.1155/2020/2529592

29. Moar, N.T. (1985). Pollen analysis of New Zealand honey. New Zealand Journal of Agricultural Research, 28(1), 3970. doi.org/10.1080/00288233.1985.10426997

30. Moore, P.D., Webb, J.A. \& Collinson, M.E. (1991). Pollen analysis. London, Blackwell Sci. Publ., ( $2^{\text {nd }}$ edition), pp. 216.

31. Neha Singh \& Chaturvedi, S.K. (2016). Melissopalynological Studies of Honey Samples from Bastar District, Chhattisgarh. The International Journal of Plant Reproductive Biology, 9(1), 37-40. doi.10.14787/ijprb.2017 9.1.3 7-40

32. Neupane, K.R., Dhakal, D.D., Thapa, R.B. \& Gautam, D.M. (2006). Foraging preference of giant honeybee Apis dorsata F., to selected horticultural crops. Journal of the Institute of Agriculture and Animal Science, 27, 87-92.

33. Novais, J.S., Lima, L.C.L. \& Santos, F.A.R. (2009). Botanical affinity of pollen harvested by Apis mellifera $\mathrm{L}$. in a semi-arid area from Bahia, Brazil. Grana, 48(3), 224234. doi:10.1080/00173130903037725

34. Oliveira, P.P., Van Den Berg, C. \& Santos, F.D.A.R.D. (2010). Pollen analysis of honeys from Caatinga vegetation of the state of Bahia, Brazil. Grana, 49(1), 66-75. doi.org/10.1080/00173130903485122

35. Raghunandan \& Basavarajappa. (2014). Melissopalyonology of multifloral honey of Asian giant honeybee Apis dorsata fabricius at Southern Karnataka, India. Indian Journal of Applied Research, 4(8), 667-669.

36. Ramalho, M. \& Kleinert-Giovannini, A. (1986). Some aspects of the utilization of pollen analysis in ecological research. Apidologie, 17(2), 159-174. doi.org/10.1051/ apido: 19860207

37. Ramirez-Arriaga, E., Navarro-Calvo, L.A. \& Diaz-Carbajal, E. (2011). Botanical characterisation of Mexican honeys from a subtropical region (Oaxaca) based on pollen analysis. Grana, 50(1), 40-54. doi.org/10.1080/001731 34.2010.537767

38. Rodopoulou, M.A., Tananaki, C., Bimou, M., Liolios, V., Kanelis, D., Goras, G. \& Thrasyvoulou, A. (2018). The determination of the botanical origin in honeys with over $\square$ represented pollen: combination of melissopalynological, sensory and physicochemical analysis. Journal of the Science of Food and Agriculture, 98(7), 27052712. doi.org/10.1002/jsfa. 8764

39. Sajwani, A., Farooq, S.A., Patzelt, A., Eltayeb, E.A. \& Bryant, V.M. (2007). Melissopalynological studies from Oman. Palynology, 31, 63-79.

40. Sen, J. \& Banerjee, D. (1956). A pollen analysis of Indian 
honey. Bee World, 37(3), 52-54. doi.org/10.1080/00057 72X.1956.11094919

41. Shubharani, R., Sivaram, V. \& Roopa, P. (2012). Assessment of honey plant resources through pollen analysis in Coorg honeys of Karnataka state. The International Journal of Plant Reproductive Biology, 4(1), 31-39.

42. Silva, A.P.C. \& Santos, F.A.R. (2014). Pollen diversity in honey from Sergipe, Brazil. Grana, 53(2), 159-170. doi:10.1080/00173134.2014.896941

43. Singh, A.K. Deepak Kumar Jaiswal, Singh, H.K. \& Thakur, R.K. (2016). Diversity of Bees' flora and floral calendar of native honey bees in Nagaland, India. Advances in Life Sciences, 5(6), 2285-2292.

44. Tamar Keasar \& Avi Shmida. (2009). An evaluation of Israeli forestry trees and shrubs as potential forage plants. Israeli Journal of Plant Sciences, 57(1), 49-64. doi:10.1560/IJPS.57.1-2.49

45. Terrab, A., Pontes, A., Heredia, F.J. \& Diez, M.J. (2004). Palynological and geographical characterization of avoca- do honeys in Spain. Grana, 43(2), 116-121. doi.org/10.1080/00173130310017634

46. Turner, V. (1984). Banksia pollen as a source of protein in the diet of two Australian marsupials Cercartetus nanus and Tarsipus rostratus. Oikos, 43, 53-61.

47. Upadhyay, D. \& Bera, S. (2012). Pollen spectra of natural honey samples from a coastal district of Orissa, India. Journal of Apicultural Research, 51(1), 10-22. doi.org/1 0.3896/IBRA.1.51.1.03

48. Vishnu-Mittre. (1958). Pollen content of some Indian honeys. Journal of Scientific and Industrial Research, 17, 123-124.

49. White, J.W. (2005). Honey. In: The hive and the honey bee, (Graham JM, ed.) Hamilton, Illinois: Dadant \& Sons. pp. 869-927

50. Yao, Y.F., Bera, S., Wang, Y.F. \& Li, C.S. (2006). Nectar and pollen source for honeybee Apis cerana cerana Fabr. during October-November in Qinglan Harbor mangrove area, Hainan Island, China. Journal of Integrative Plant Biology, 11(48), 1266-1273. 